\title{
Optical Frequency Comb Generation Using CMOS Compatible Cascaded Mach-Zehnder Modulators
}

\author{
Zifei Wang, Ming Ma, Hao Sun, Mostafa Khalil, Rhys Adams, Kathy Yim, Xian Jin, \\ and Lawrence R. Chen, Senior Member, IEEE
}

\begin{abstract}
Optical frequency combs (OFCs) play a key role in a variety of applications and have been implemented primarily using mode-locked lasers, Kerr resonators, and electro-optic (EO) modulation. EO modulation-based OFC generation may not yield as many comb lines nor span as broad a bandwidth as modelocked lasers or Kerr resonators; however, it offers a high degree of tunability in central frequency and comb spacing. Integrated solutions are of interest as they significantly reduce device footprint and enable large scale system integration. In this paper, we demonstrate flexible on-chip OFC generation using two cascaded EO Mach-Zehnder modulators in silicon photonics. We demonstrate quasi-rectangular OFCs with 9 lines and a comb spacing of up to $10 \mathrm{GHz}$ with an amplitude variation (comb flatness) within $6.5 \mathrm{~dB}$. The corresponding time-domain waveforms have a good fit with sinc-shaped Nyquist pulses having a full-width at half maximum duration as short as 11.4 ps.
\end{abstract}

Index Terms - Optical frequency comb generation, electro-optic Mach-Zehnder modulators, silicon photonics.

\section{INTRODUCTION}

$\mathrm{O}$ PTICAL frequency combs (OFCs), which consist of a series of equidistant spectral lines with correlated phase [1], have been used extensively in a diverse range of applications including spectroscopy [2,3], optical frequency metrology [4], precision distance measurement [5], optical and microwave waveform synthesis [6-9], astronomical spectrograph calibration [10, 11], and optical communications [12]. Techniques for OFC generation include, amongst others, the use of mode-lock lasers assisted by an internal or external reference [13-20], Kerr resonators [21-30], and electro-optic (EO) modulation [31-37]. The properties of mode-locked lasers, such as repetition rate, amplitude noise, linewidth, and frequency stability determine the characteristics of the OFC and need to be considered carefully for different applications [18]. By exploiting parametric four-wave mixing, OFC generation based on Kerr resonators may offer octave-spanning, ultrawide bandwidth from a single continuous wave (CW) input, as well

Submitted 21 June 2019. This work was supported in part by the Natural Sciences and Engineering Research Council of Canada and the Fonds de Recherche du Québec - Nature et Technologies.

The authors are with the Department of Electrical and Computer Engineering, McGill University, Montreal, QC H3A 0E9 Canada (emails: as high frequency and timing stability, which are desirable for telecommunications, spectroscopy, low-noise microwave waveform synthesis, and distance ranging [27]. EO modulationbased OFC generation offers the flexibility to tune readily the center frequency and comb spacing simply by adjusting the input $\mathrm{CW}$ wavelength as well as frequency, power, and phase of the RF signal(s) applied to the EO modulators. Cascading a larger number of modulators (e.g., $\geq 3$ ) is often used to increase the number of comb lines and obtain a broadband comb [8], though it may not yield as many comb lines or span as broad a bandwidth as mode-locked lasers or Kerr resonators. In some applications, e.g., the generation of sinc-shaped Nyquist pulses for Nyquist orthogonal time-division multiplexed transmission, the shape of the comb spectrum is more important than obtaining a large number of comb lines. In [35], two modulators are cascaded to produce a rectangular shaped, phase-locked comb with 9 to 10 spectral lines occupying more than $100 \mathrm{GHz}$ bandwidth and corresponding high quality Nyquist optical pulses.

For still other applications in optical communications and microwave photonics, a high degree of system level integration may be more desirable over a large number of comb lines. To date, integrated OFC generators have been reported using various technology platforms such as silica [21, 22], Hydex glass [23], silicon nitride ( $\mathrm{SiN}$ ) [24-27], III-V semiconductors [28, 29], silicon-organic hybrids [36], and silicon-on-insulator (SOI) $[32,37,38]$. Exploiting recent advances in developing high-performance EO modulators in SOI has the potential to yield a highly-tunable integrated comb source.

In this paper, we demonstrate the generation of an EO modulation-based OFC using integrated cascaded push-pull traveling-wave Mach-Zehnder modulators (MZMs) in SOI. By precisely controlling the amplitude and phase of the driving RF signals as well as the bias and thermal heating voltages, a quasirectangular OFC with 9 lines, flatness within $6.5 \mathrm{~dB}$, and bandwidths up to $90 \mathrm{GHz}$ have been obtained; the corresponding sinc-shaped Nyquist pulses have a full-width at

\{zifei.wang, ming.ma, hao.sun3, mostafa.khalil2, kathy.yim, xian.jin\}@mail.mcgill.ca, lawrence.chen@mcgill.ca).

M. Ma is now with Ericsson Canada Inc. 8275 Trans Canada Route, SaintLaurent, QC H4S 0B6 Canada.

R. Adams is also with the Department of Physics, CEGEP Vanier College, Montreal, QC H4L 3X9 Canada (email: adamsr@vanier.college). 
half-maximum (FWHM) duration as short as 11.4 ps.

\section{DEVICE DESIGN}

Figures 1 (a) and (b) show a schematic of the two cascaded EO MZMs in SOI and a photo of the fabricated device. The modulators are based on the series push-pull design described in [39]; some of the parameters are reviewed here. The devices are fabricated on a $220 \mathrm{~nm}$ SOI wafer with a $750 \Omega-\mathrm{cm}$ silicon substrate using a CMOS-compatible foundry process available at IME A*STAR. Rib waveguides are doped using three doping levels to form diode waveguides; their crosssections are shown in Fig. 1(c). The waveguides sit on top of a $3 \mu \mathrm{m}$ thick oxide (BOX) and are covered by a $2 \mu \mathrm{m}$ thick index matched oxide cladding. The waveguide cross-sections and doping parameters are given in Table 1 .

Each modulator has a path imbalance of $130 \mu \mathrm{m}$, which introduces an additional phase shift in the lower arm of the MZ interferometer and creates a periodic spectral response. This path imbalance is used to measure the phase shifts associated with bias and thermal tuning, as described below. An n++ doped heater with a length of $215 \mu \mathrm{m}$ is overlaid on the upper arm for fine-tuning the phase. The length of each modulator is $4.55 \mathrm{~mm}$ and the net $\mathrm{p}-\mathrm{n}$ junction loading is $4.2 \mathrm{~mm}$. The two modulators are connected in series using $2 \times 2$ MMI couplers [40] and in order to characterize each modulator separately, additional taps are used. Vertical grating couplers (VGCs) designed for TE mode operation over the $\mathrm{C}$ band [41] serve for input and output coupling and testing. The total size of the device is $1.49 \mathrm{~mm} \times 5.3 \mathrm{~mm}$.

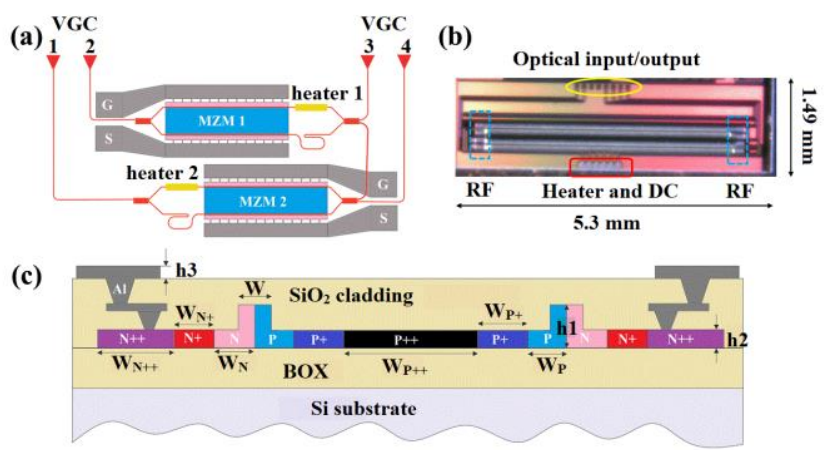

Fig. 1. Integrated OFC generator in SOI: (a) device schematic, (b) photo of fabricated device, and (c) cross-section of push-pull MZM.

TABLE I

PARAMETERS OF THE PUSH-PULL EO MZMS IN SOI

\begin{tabular}{|c|c|}
\hline $\mathrm{h} 1$ & $220 \mathrm{~nm}$ \\
\hline $\mathrm{h} 2$ & $90 \mathrm{~nm}$ \\
\hline $\mathrm{h} 3$ & $2 \mu \mathrm{m}$ \\
\hline $\mathrm{W}$ & $500 \mathrm{~nm}$ \\
\hline $\mathrm{W}_{\mathrm{N}++}$ & $5.2 \mu \mathrm{m}$ \\
\hline $\mathrm{W}_{\mathrm{N+}}$ & $0.81 \mu \mathrm{m}$ \\
\hline $\mathrm{W}_{\mathrm{N}}$ & $0.39 \mu \mathrm{m}$ \\
\hline $\mathrm{W}_{\mathrm{P}++}$ & $28 \mu \mathrm{m}$ \\
\hline $\mathrm{W}_{\mathrm{P}+}$ & $0.83 \mu \mathrm{m}$ \\
\hline $\mathrm{W}_{\mathrm{P}}$ & $0.37 \mu \mathrm{m}$ \\
\hline
\end{tabular}

\section{EXPERIMENTS AND RESULTS}

\section{A. Characterization of the MZMs}

First, we characterize each MZM separately. Figure 2(a) shows the transmission spectra of MZM 1 and MZM 2 as a function of reverse (DC) bias voltage. The fiber-to-fiber loss (i.e., static loss in the absence of any applied RF signal) depends on operating wavelength and bias/heating voltages. When measuring the loss of each MZM, we use a wavelength that matches with a peak in the corresponding spectral response without applied voltages. MZM 1 has a fiber-to-fiber insertion loss (i.e., from $\mathrm{VGC} 2$ to $\mathrm{VGC} 3$ ) of $18 \mathrm{~dB}$ at a wavelength of $1548 \mathrm{~nm}$; this comprises $11 \mathrm{~dB}$ coupling loss, $4 \mathrm{~dB}$ loss from the MMI, and $3 \mathrm{~dB}$ loss from the MZM structure itself. MZM 2 also has a fiber-to-fiber insertion loss (i.e., from VGC4 to VGC1) of $18 \mathrm{~dB}$, but at a wavelength of $1550 \mathrm{~nm}$. The total fiber-to-fiber insertion loss of the cascaded MZMs (i.e., from VGC2 to VGC1) is $24 \mathrm{~dB}$ at a wavelength of $1550 \mathrm{~nm}$. When no bias voltage is applied, the extinction ratios at a wavelength of $1550 \mathrm{~nm}$ for MZM 1 and MZM 2 are $30 \mathrm{~dB}$ and $19.5 \mathrm{~dB}$, respectively. By increasing the bias voltage, the difference in carrier concentration between the two arms of the MZM increases, which induces a higher optical loss imbalance and the degradation in extinction ratio. Both MZMs have a free spectral range of $4.4 \mathrm{~nm}$, which corresponds to the designed path imbalance of $130 \mu \mathrm{m}$. The half-wave voltages of both MZMs are $V_{\pi}=10 \mathrm{~V}$, indicating $V_{\pi} L=4.2 \mathrm{~V}-\mathrm{cm}$. The travellingwave MZM design results in a relatively high value of $V_{\pi}$; in order to reduce $V_{\pi}$, the doping length must be longer, which will increase the propagation loss and reduce bandwidth. Figure 2(b) shows the transmission spectrum of MZM 1 and MZM 2 as a function of heating voltage (i.e., voltage applied to heater 1 or heater 2). In this case, both MZMs attain a $\pi$ shift for a heating voltage of $2.5 \mathrm{~V}$. Therefore, we can use the heating voltage and DC bias for coarse and fine phase tuning of the modulator operation.
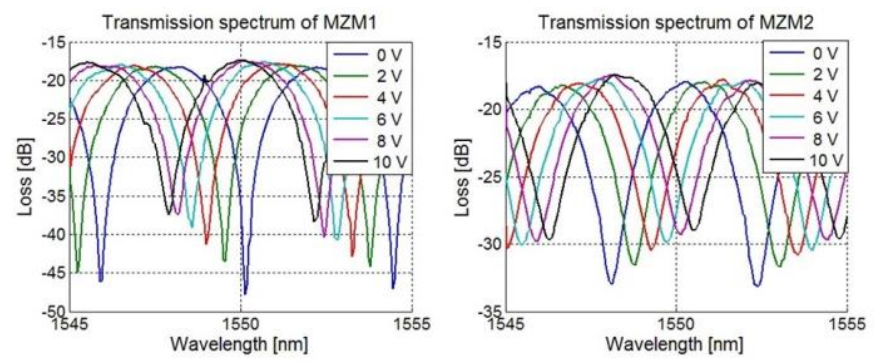

(a)
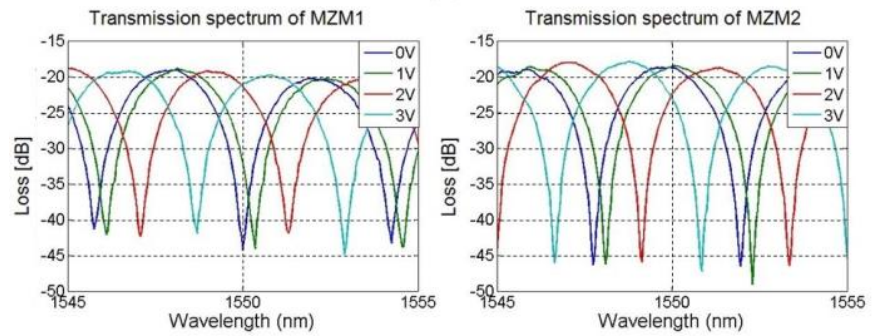

(b)

Fig. 2. Measured transmission spectra of MZM 1 and MZM 2 for various (a) reverse bias voltages and (b) heating voltages. 
Figure 3 shows the measured $S$ parameters of both MZMs. For a reverse bias of $3 \mathrm{~V}$, the $3 \mathrm{~dB}(6 \mathrm{~dB})$ bandwidths of MZM 1 and MZM 2 are $13.1 \mathrm{GHz}(25 \mathrm{GHz})$ and $10.9 \mathrm{GHz}(22.8$ $\mathrm{GHz}$ ), respectively. Note that the $S_{11}$ value is below $-10 \mathrm{~dB}$ for frequencies up to $50 \mathrm{GHz}$, indicating good $50 \Omega$ matching.
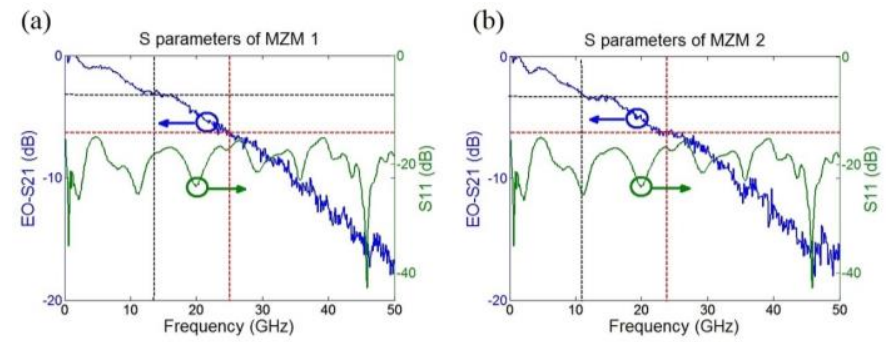

Fig. 3. Measured $S_{1 I}$ and $S_{2 l}$ parameters of (a) MZM 1 and (b) MZM 2.

(a)

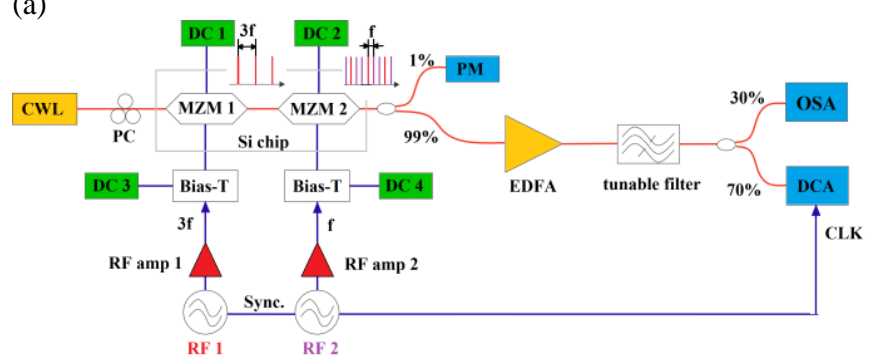

(b)

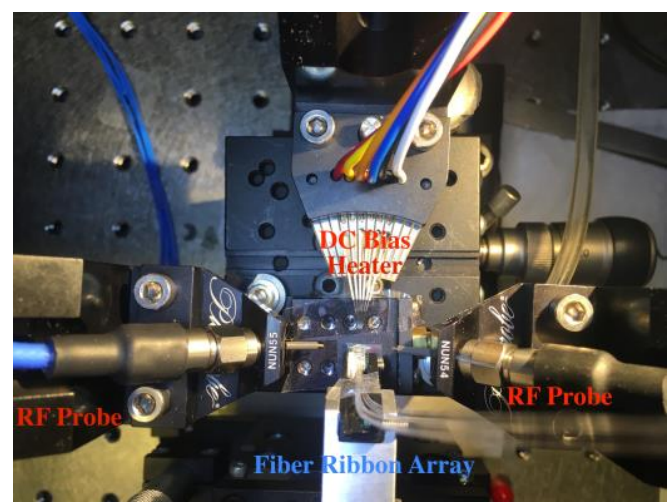

Fig. 4. (a) Schematic and (b) photo of experimental setup for on-chip OFC generation. CWL: continuous wave laser, RF: radio frequency, DC: direct current, PM: power meter, EDFA: erbium-doped fiber amplifier, OSA: optical spectrum analyzer, DCA: digital communications analyzer.

\section{B. OFC generation}

Figures 4(a) and 4(b) show a schematic and photo of our experimental setup for on-chip OFC generation. A CW laser at $1550.02 \mathrm{~nm}$ with a power of $14.2 \mathrm{dBm}$ is used as a seed carrier for MZM 1. By tuning the amplitude of the RF signal (RF 1), as well as the DC reverse bias and heater voltages of MZM 1, we can obtain two first-order sidebands with approximately the same power as the seed carrier while suppressing the higherorder sidebands. These three spectral lines (which are separated by the frequency of RF 1) are then injected into MZM 2 (driven by a second RF signal, RF 2) as new seed carriers to generate additional first-order sidebands; the final result is a total of 9 comb lines. In order to generate combs with equal frequency spacing, the frequency of RF 2 should be $1 / 3$ that of RF 1 .
Moreover, the two RF signals should be synchronized properly by tuning their phases (especially for optimizing the quality of the sinc-shaped Nyquist pulses). The OFC at the output of the chip is amplified using an EDFA and filtered using a tunable filter (Finisar Waveshaper) to suppress out-of-band amplified spontaneous emission noise. The spectra and temporal waveforms are measured using an optical spectrum analyzer (OSA) with a resolution of $10 \mathrm{pm}$ and an optical sampling module connected to a digital sampling oscilloscope with an impulse response time of $6.8 \mathrm{ps}$. The oscilloscope is operated in sample mode (no averaging) with a persistence time of 100 ms.
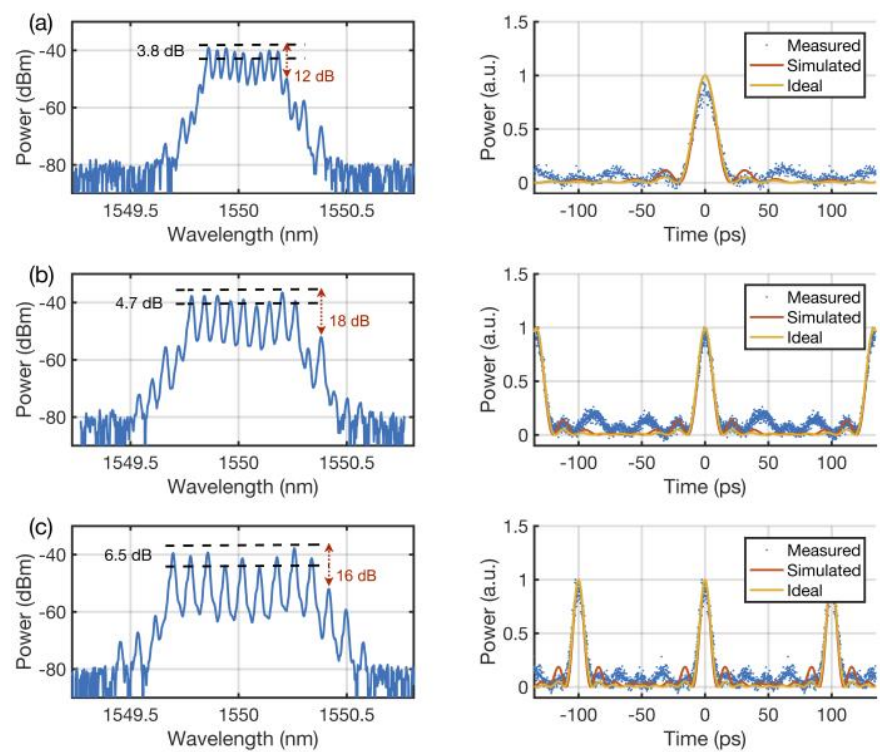

Fig. 5. OFC spectra at the output of the cascaded modulators (left) and corresponding temporal waveforms (right) for a comb spacing of (a) $5 \mathrm{GHz}$, (b) $7.5 \mathrm{GHz}$, and (c) $10 \mathrm{GHz}$. All temporal waveforms are obtained without averaging. The zero crossing pulse durations are $44.3 \mathrm{ps}, 28.7 \mathrm{ps}$, and $25.2 \mathrm{ps}$, for (a), (b), and (c), respectively, and the corresponding FWHM pulse durations are $17.6 \mathrm{ps}, 13.1 \mathrm{ps}$, and 11.4.

Figure 5 shows the spectra and the corresponding temporal waveforms of the OFC. In the frequency domain, we can control the flatness of the combs by adjusting the RF power and tuning the heater voltages of the MZMs (in our experiments, we set the DC reverse bias voltages to 0 The range of the heater voltages used is between $2.8 \mathrm{~V}$ to $3.8 \mathrm{~V}$. The power of the RF signals after amplification (i.e., RF amp 1 and RF amp 2) are $11 \mathrm{dBm}$ and $14.5 \mathrm{dBm}$ for RF 1 (higher frequencies) and RF 2 (lower frequencies), respectively. Once the 9 comb lines are generated, the temporal waveforms are optimized further by tuning the phases of the RF signals. The spectra shown in Fig. 5 are for comb spacings of $5 \mathrm{GHz}, 7.5 \mathrm{GHz}$, and $10 \mathrm{GHz}$ and are measured before the EDFA. The corresponding powers at the output of the chip are $-28.6 \mathrm{dBm},-26.8 \mathrm{dBm}$, and $-28.5 \mathrm{dBm}$ and the quasi-rectangular shaped combs have a flatness within $3.8 \mathrm{~dB}, 4.7 \mathrm{~dB}$, and $6.5 \mathrm{~dB}$, and a sideband suppression of 12 $\mathrm{dB}, 18 \mathrm{~dB}$, and $16 \mathrm{~dB}$. Finally, the optical signal to out-of-band noise for the comb lines is $\sim 40 \mathrm{~dB}$; future work includes transmission system experiments so that we can assess the performance of our generated OFCs for Nyquist orthogonal 
time-division multiplexed and WDM transmission.

To obtain 3 comb lines with roughly equal power (e.g., from the first modulator), we need to suppress intentionally the power of the carrier to match that of the first-order sidebands. Since the $3 \mathrm{~dB}$ bandwidths of the two MZMs are in the range of $10.9 \mathrm{GHz}$ to $13.1 \mathrm{GHz}$, it is easier to obtain 3 comb lines with less power variation at lower frequencies; on the other hand, when we increase the operating frequency, e.g., to $10 \mathrm{GHz}$ which requires on $\mathrm{RF}$ signal at $30 \mathrm{GHz}$ ), achieving flatness over the 9 comb lines requires higher RF power and greater variation in the heater voltages. Each of the OFC spectra shown in Fig. 5 was optimized separately and this optimization process created the differences in comb flatness. The need to suppress the power of the carrier contributes to a higher dynamic insertion loss (i.e., when an RF signal is applied to the modulators) of $\sim 43 \mathrm{~dB}$ (compared to the static insertion loss of $18 \mathrm{~dB}$ described earlier).
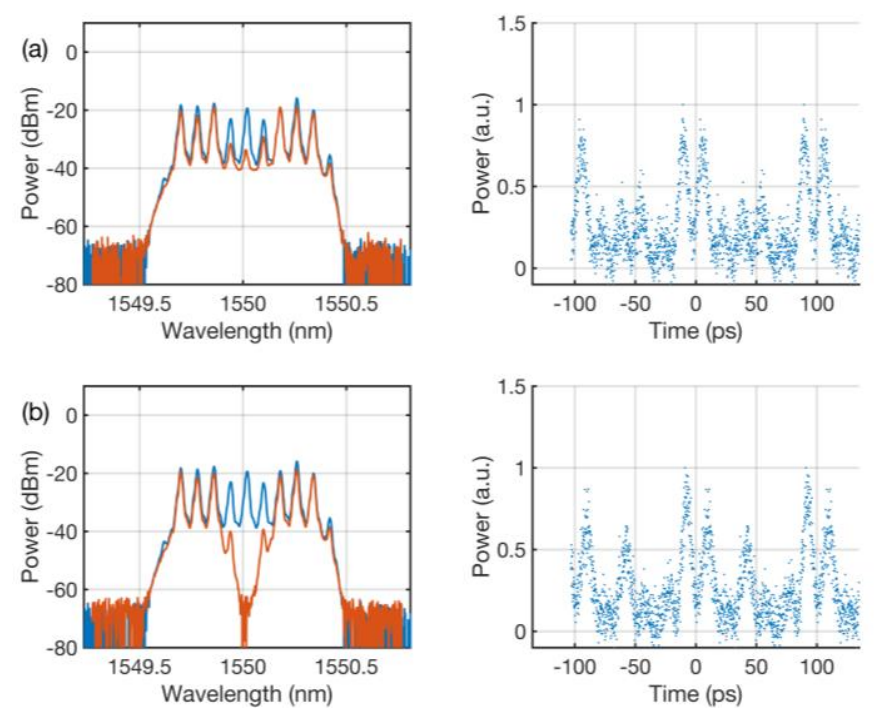

Fig. 6. Waveform shaping: spectra (left) and temporal waveforms (right) when the 3 central comb lines are removed using (a) fine tuning of the heater voltages and (b) a 'notch' filter.

The measured temporal waveforms are in excellent agreement with ideal sinc-shaped Nyquist pulses (assuming a perfect rectangular shaped OFC) and the simulated pulses (using the measured amplitudes of the comb lines). The sincshaped Nyquist pulses have FWHM durations of $17.6 \mathrm{ps}, 13.1$ ps, and 11.4 ps and the corresponding rms timing jitters are 1.2 ps, $1.7 \mathrm{ps}$, and $1.1 \mathrm{ps}$ (all timing jitter measurements were made using the digital sampling oscilloscope with a precision time base and a persistence time of $100 \mathrm{~ms}$ ). The RF synthesizers used in our experiments have rms timing jitters below $300 \mathrm{fs}$. The system (electrical and optical components such as RF amplifiers and EDFA) as well as operating parameters (e.g., synchronization of the applied RF signals to the two MZMs) contribute to an increase in the rms timing jitter of the output Nyquist pulses. The measured rms timing jitters are nevertheless representative of high-quality optical signals, including at $10 \mathrm{~Gb} / \mathrm{s}$. Note that due to some phase mismatching between the two RF signals, there is a small difference in the sidelobes between the measured and simulated waveforms; we believe that we can minimize these sidelobes with further optimization of the RF phases.

We can also 'shape' the comb and corresponding temporal waveform by tuning the heater voltages or using a tunable optical filter. For example, in Fig. 6, we suppress the three middle comb lines by adjusting the heater voltages [6(a)] or using the Waveshaper to implement a notch-like filter [6(b)]. The ability to tailor the comb shape finely allows for the generation of more complex waveforms such as optical pulse bursts, triangular pulses, etc. [6, 42, 43].

\section{SUMMARY AND DISCUSSION}

We have demonstrated on-chip generation of quasirectangular OFCs using two cascaded push-pull traveling-wave EO MZMs in SOI. We obtained OFCs with a comb spacing of up to $10 \mathrm{GHz}$ (OFC bandwidth of $90 \mathrm{GHz}$ ); the flatness varies from $3.8 \mathrm{~dB}$ to $6.5 \mathrm{~dB}$ and the sideband suppression ranges from $12 \mathrm{~dB}$ to $16 \mathrm{~dB}$. The corresponding temporal sinc-shaped Nyquist pulses have FWHM pulse durations as short as $11.4 \mathrm{ps}$ and with rms timing jitters below 1.7 ps.

While OFCs generated using cascaded $\mathrm{LiNbO}_{3}$ modulators (e.g., [35]) can achieve greater comb flatness (within $0.2 \mathrm{~dB}$ ), higher sideband suppression $(27 \mathrm{~dB})$, and lower timing jitter (< $100 \mathrm{fs}$ ), our results demonstrate the potential for what can be obtained with on-chip comb generation and further improvements may be possible. For example, we can increase the available output power (i.e., reducing the dynamic insertion loss) by using VGCs with lower coupling loss [44] as well as having a higher MZM bandwidth and/or reducing $V_{\pi}$ (both of these will reduce the amount of carrier suppression required to equalize the carrier and sideband power levels). MZM bandwidths of $41 \mathrm{GHz}$ have been demonstrated [39]; thus, it should be possible to obtain 9 comb lines with a spacing of 15 $\mathrm{GHz}$ (corresponding to a total bandwidth of $135 \mathrm{GHz}$ ). While careful design may reduce $V_{\pi}$ further, the typical values in SOI are larger compared to $\mathrm{LiNbO}_{3}$ and as such, it may be difficult to obtain more than 9 lines. However, the integrated OFC generator can be used as a source for Nyquist orthogonal timedivision multiplexed transmission $[35,45,46]$ or flexible WDM transmission where flexibility in terms of comb spacing and modulation format is more important than a larger number of comb lines [37].

We believe that our on-chip OFC generator can be used as a building block for developing more advanced integrated subsystems for applications in optical communications, e.g., as an on-chip transmitter, and microwave photonics, e.g., microwave photonic filters and RF channelization.

\section{ACKNOWLEDGMENT}

We thank D. Patel for assistance in device layout, M. Jacques for assistance in $S$-parameter measurements, D. V. Plant for providing a number of components used in the experiments, and CMC Microsystems and the NSERC CREATE Si-EPIC program.

\section{REFERENCES}


[1] S. T. Cundiff and J. Ye, "Colloquium: Femtosecond optical frequency combs," Rev. Mod. Phys., vol. 75, no. 1, pp.325-342, Mar 2013.

[2] M.-G. Suh, Q.-F. Yang, K. Y. Yang, X. Yi, and K. J. Vahala, "Microresonator soliton dual-comb spectroscopy," Science, vol. 354, no. 6312, pp. 600-603, Nov 2016.

[3] C. Gohle, T. Udem, M. Herrmann, J. Rauschenberger, R. Holzwarth, H. A. Schuessler, F. Krausz, and T. W. Hänsch, "A frequency comb in the extreme ultraviolet," Nature, vol. 436, no. 7048, pp. 234-237, Jul 2005.

[4] T. Udem, R. Holzwarth, and T. W. Hänsch, "Optical frequency metrology," Nature, vol. 416, no. 6877, pp. 233-237, Mar 2002.

[5] M.-G. Suh, and K. J. Vahala, "Soliton microcomb range measurement," Science, vol. 359, no. 6378, pp. 884-887, Feb 2018.

[6] C.-B. Huang, Z. Jiang, D. Leaird, J. Caraquitena, and A. M. Weiner, "Spectral line-by-line shaping for optical and microwave arbitrary waveform generations," Laser Photonics Rev., vol. 2, no. 4, pp. 227-248, Aug 2008.

[7] D. T. Spencer, S. H. Lee, D. Y. Oh, M.-G. Suh, K. Y. Yang, and K. J. Vahala, "An optical-frequency synthesizer using integrated photonics," Nature, vol. 557, no. 7703, pp. 81-85, May 2018.

[8] V. Torres-Company and A. M. Weiner, "Optical frequency comb technology for ultra-broadband radio-frequency photonics," Laser Photonics Rev., vol. 8, no. 3, pp. 368-393, Dec 2013.

[9] W. Liang, D. Eliyahu, V. S. Ilchenko, A. A. Savchenkov, A. B. Matsko, D. Seidel, and L. Maleki, "High spectral purity Kerr frequency comb radio frequency photonic oscillator," Nat. Commun., vol. 6, pp. 7957, Aug. 2015.

[10] C.-H. Li, A. J. Benedick, P. Fendel, A. G. Glenday, F. X. Kärtner, D. F. Phillips, D. Sasselov, A. Szentgyorgyi, R. L. Walsworth, "A laser frequency comb that enables radial velocity measurements with a precision of $1 \mathrm{~cm} \mathrm{~s}-1, "$ Nature, vol. 452, pp. 610-612, Apr 2008.

[11] T. Steinmetz, T. Wilken, C. Araujo-Hauck, R. Holzwarth, T. W. Hänsch, L. Pasquini, A. Manescau, S. D’Odorico, M. T. Murphy, T. Kentischer, W. Schmidt, and T. Udem, "Laser frequency combs for astronomical observations," Science, vol. 321, no. 5894, pp.1335-1337, Sep 2008.

[12] P. Marin-Palomo, J. N. Kemal, M. Karpov, A. Kordts, J. Pfeifle, M. H. P. Pfeiffer, P. Trocha, S. Wolf, V. Brasch, M. H. Anderson, R. Rosenberger, K. Vijayan, W. Freude, T. J. Kippenberg, and C. Koos, "Microresonatorbased solitons for massively parallel coherent optical communications," Nature, vol. 546, no. 7657, pp. 274-279, Jun 2017.

[13] D. J. Jones, S. A. Diddams, J. K. Ranka, A. Stentz, R. S. Windeler, J. L. Hall, and S. T. Cundiff, "Carrier-envelope phase control of femtosecond mode-locked lasers and direct optical frequency synthesis," Science, vol. 288, no. 5466, pp.635-639, Apr 2000.

[14] B. R. Washburn, S. A. Diddams, N. R. Newbury, J. W. Nicholson, M. F. Yan, and C. G. Jørgensen, "Phase-locked, erbium-fiber-laser-based frequency comb in the near infrared," Opt. lett., vol. 29 , no. 3, pp. 250252, Feb 2004

[15] A. Bartels, D. Heinecke, and S. A. Diddams, "10-GHz self-referenced optical frequency comb," Science, vol. 326, no. 5953, pp. 681-681, Oct 2009.

[16] S. A. Diddams, "The evolving optical frequency comb," J. Opt. Soc. Am. B: Opt. Phys., vol. 27, no. 11, pp. B51-B62, Nov 2010.

[17] D. Burghoff, T.-Y. Kao, N. Han, C. W. I. Chan, X. Cai, Y. Yang, D. J. Hayton, J.-R. Gao, J. L. Reno, and Q. Hu, "Terahertz laser frequency combs." Nat. Photonics, vol. 8, no. 6, pp. 462-467, May 2014.

[18] J. Kim and Y. Song, "Ultralow-noise mode-locked fiber lasers and frequency combs: principles, status, and applications," Adv. Opt. Photonics, vol. 8, no. 3, pp. 465-540, 2016.

[19] S. Uvin, S. Keyvaninia, F. Lelarge, G.-H. Duan, B. Kuyken, and G. Roelkens, "Narrow line width frequency comb source based on an injection-locked III-V-on-silicon mode-locked laser," Opt. Express, vol. 24, no. 5, pp. 5277-5286, Mar 2016.

[20] Z. Wang, K. V. Gasse, V. Moskalenko, S. Latkowski, E. Bente, B. Kuyken, and G. Roelkens, "A III-V-on-Si ultra-dense comb laser," Light Sci. Appl., vol. 6, no. 5, pp. e16260, May 2017.

[21] J. Li, H. Lee, T. Chen, and K. J. Vahala, "Low-pump-power, low-phasenoise, and microwave to millimeter-wave repetition rate operation in microcombs," Phys. Rev. Lett., vol. 109, no. 23, pp. 233901, Aug 2012.

[22] P. Del'Haye, A. Schliesser, O. Arcizet, T. Wilken, R. Holzwarth, and T. J. Kippenberg, "Optical frequency comb generation from a monolithic microresonator," Nature, vol. 450, no. 7173, pp. 1214-1217, Dec 2007.

[23] L. Razzari, D. Duchesne, M. Ferrera, R. Morandotti, S. Chu, B. E. Little, and D. J. Moss, "CMOS-compatible integrated optical hyper-parametric oscillator," Nat. Photonics, vol. 4, no. 1, pp. 41-45, Dec 2009.
[24] J. S. Levy, A. Gondarenko, M. A. Foster, A. C. Turner-Foster, A. L. Gaeta, and M. Lipson, "CMOS-compatible multiple-wavelength oscillator for on-chip optical interconnects," Nat. Photonics, vol. 4, no. 1, pp. 37-40, Dec 2009

[25] T. J. Kippenberg, R. Holzwarth, and S. A. Diddams, "Microresonatorbased optical frequency combs," Science, vol. 332, no. 6029, pp.555-559, Apr 2011

[26] P. Trocha, M. Karpov, D. Ganin, M. H. P. Pfeiffer, A. Kordts, S. Wolf, J. Krockenberger, P. Marin-Palomo, C. Weimann, S. Randel, W. Freude, T. J. Kippenberg, and C. Koos, "Ultrafast optical ranging using microresonator soliton frequency combs," Science, vol. 359, no. 6378, pp. 887-891, Feb 2018.

[27] B. Stern, X. Ji, Y. Okawachi, A. L. Gaeta, and M. Lipson, "Batteryoperated integrated frequency comb generator," Nature, vol. 562, no. 7727, pp. 401-405, Oct 2018.

[28] N. Dupuis, C. R. Doerr, L. Zhang, L. Chen, N. J. Sauer, P. Dong, L. L. Buhl, and D. Ahn, "InP-based comb generator for optical OFDM," $J$. Lightwave Technol., vol. 30, no. 4, pp. 466-472, Oct 2011.

[29] M. Pu, L. Ottaviano, E. Semenova, and K. Yvind, "Efficient frequency comb generation in AlGaAs-on-insulator," Optica, vol. 3, pp. 823-826, Jul 2016

[30] B. Kuyken, T. Ideguchi, S. Holzner, M. Yan, T. W. Hänsch, J. V. Campenhout, P. Verheyen, S. Coen, F. Leo, R. Baets, G. Roelkens, and N. Picqué, "An octave-spanning mid-infrared frequency comb generated in a silicon nanophotonic wire waveguide," Nat. Commun., vol. 6, pp. 6310, Feb 2015

[31] T. Sakamoto, T. Kawanishi, and M. Izutsu, "Asymptotic formalism for ultraflat optical frequency comb generation using a Mach-Zehnder modulator," Opt. Lett., vol. 32, no. 11, pp. 1515-1517, May 2007.

[32] I. L. Gheorma, and G. K. Gopalakrishnan, "Flat frequency comb generation with an integrated dual-parallel modulator," IEEE Photonics Technol. Lett., vol. 19, no. 13, pp. 1011-1013, Jun 2007.

[33] Z. Jiang, C.-B. Huang, D. E. Leaird, and A. M. Weiner, "Optical arbitrary waveform processing of more than 100 spectral comb lines," Nat. Photonics, vol. 1, no. 8, pp. 463-467, Aug 2007.

[34] R. Wu, V. R. Supradeepa, C. M. Long, D. E. Leaird, and A. M. Weiner, "Generation of very flat optical frequency combs from continuous-wave lasers using cascaded intensity and phase modulators driven by tailored radio frequency waveforms," Opt. Lett., vol. 35, no. 19, pp. 3234-3236, Oct 2010.

[35] M. A. Soto, M. Alem, M. A. Shoaie, A. Vedadi, C.-S. Brès, L. Thévenaz, and T. Schneider, "Optical sinc-shaped Nyquist pulses of exceptional quality." Nat. Commun. vol. 4, pp. 2898, Dec 2013.

[36] C. Weimann, P. C. Schindler, R. Palmer, S. Wolf, D. Bekele, D. Korn, J. Pfeifle, S. Koeber, R. Schmogrow, L. Alloatti, D. Elder, H. Yu, W. Bogaerts, L. R. Dalton, W. Freude, J. Leuthold, and C. Koos, "Siliconorganic hybrid $(\mathrm{SOH})$ frequency comb sources for terabit/s data transmission," Opt. Express, vol. 22, no. 3, pp. 3629-3637, Feb 2014.

[37] J. Lin, H. Sepehrian, Y. Xu, L. A. Rusch, and W. Shi, "Frequency comb generation using a CMOS compatible SiP DD-MZM for flexible networks," IEEE Photonics Technol. Lett., vol. 30, no. 17, pp. 1495-1498, Jul 2018.

[38] I. Demirtzioglou, C. Lacava, K. RH Bottrill, D. J. Thomson, G. T. Reed, D. J. Richardson, and P. Petropoulos, "Frequency comb generation in a silicon ring resonator modulator," Opt. Express, vol. 26, no. 2, pp. 790796, Jan 2018.

[39] D. Patel, S. Ghosh, M. Chagnon, A. Samani, V. Veerasubramanian, M. Osman, and D. V. Plant, "Design, analysis, and transmission system performance of a $41 \mathrm{GHz}$ silicon photonic modulator, "Opt. Express, vol. 23, no. 11, pp. 14263-14287, May 2015.

[40] D. Dai and S. He, "Optimization of ultracompact polarization-insensitive multimode interference couplers based on Si nanowire waveguides," IEEE Photonics Technol. Lett., vol. 18, no. 19, pp. 2017-2019, Oct 2006.

[41] A. Mekis, S. Gloeckner, G. Masini, A. Narasimha, T. Pinguet, S. Sahni, and P. Dobbelaere, "A grating-coupler-enabled CMOS photonics platform, " IEEE J Sel. Top. Quant., vol. 17, no. 3, pp. 597-608, Jun 2011.

[42] X. Zhou, X. Zheng, H. Wen, H. Zhang, Y. Guo, and B. Zhou, "All optical arbitrary waveform generation by optical frequency comb based on cascading intensity modulation," Opt. Commun., vol. 284, no. 15, pp. 3706-3710, Jul 2011

[43] F. Zhang, J. Wu, Y. Li, and J. Lin, "Flat optical frequency comb generation and its application for optical waveform generation," Opt. Commun., vol. 290, pp. 37-42, Mar 2013. 
[44] Y. Ding, C. Peucheret, H. Ou, and K. Yvind, "Fully etched apodized grating coupler on the SOI platform with $-0.58 \mathrm{~dB}$ coupling efficiency, " Opt. Lett., vol. 39, no. 18, pp. 5348-5340, Sep 2014.

[45] M. Nakazawa, H. Toshihiko, R. Peng, and G. Pengyu, "Ultrahigh-speed "orthogonal" TDM transmission with an optical Nyquist pulse train," Opt. Express, vol. 20, no. 2, pp. 1129-1139, Jan 2012.

[46] H. N. Tan, T. Inoue, and S. Namiki, "Pass-drop operations of $4 \times 172 \mathrm{~Gb} / \mathrm{s}$ Nyquist OTDM-WDM over cascaded of WSSs using distributed matched filtering," Prof. Conf. on Opt. Fiber Comm., paper JW2A.50, 2013.

Zifei Wang received her B.A.Sc. in electrical engineering from Nankai University, Tianjin, China, in 2011, M.Eng. in electrical engineering from Tianjin University, Tianjin, China, in 2014, and Ph.D. in electrical and computer engineering from McGill University, Montreal, QC, Canada in 2019. Her research interests include passive and active silicon photonic devices for optical communication systems.

Ming Ma received his B.Eng. in electrical engineering from Beijing Institute of Technology, Beijing, China, M.Sc. in electrical engineering from Technical University of Denmark, Denmark, and Ph.D. in electrical and computer engineering from McGill University, Montreal, QC, Canada. He is currently with Ericsson Canada Inc.

Hao Sun received his B.S. in physics from Shandong University in 2015 and M.Eng. from the Institute of Semiconductors, Chinese Academy of Sciences, Beijing, China in 2018. He is currently working toward the Ph.D. degree in electrical and computer engineering at McGill University, Montreal, QC, Canada. His research interests include silicon photonics, optical communications, and microwave photonics.

Mostafa Khalil received his B.A.Sc. in electrical engineering communications and electronics from Alexandria University, Alexandria, Egypt in 2018. Since his last year in University, he has been working on research in the field of optical communications. He is currently pursuing his M.Eng. in electrical and computer engineering at McGill University, Montreal, QC, Canada.

Rhys Adams received his B.Eng. and M.Eng. in electrical and computer engineering from McGill University, Montreal, QC, Canada in 2002 and 2005 respectively. He has been with the Department of Physics at CEGEP Vanier College since 2006. His research interests include optical communications, microwave photonics, and innovative pedagogies for physics education.

Kathy Yim is completing her B.Eng. in electrical and computer engineering at McGill University, Montreal, QC, Canada. Currently, she is working on applications of silicon photonics devices.
Xian Jin received his B.Eng. degree in electronic and information engineering from the Nanjing University of Science and Technology, Nanjing, China, in 2006, and his M.A.Sc. and $\mathrm{Ph}$. D. degrees in electrical engineering from the University of British Columbia, Kelowna, BC, Canada, in 2009 and 2016, respectively. He is currently a postdoctoral researcher in electrical and computer engineering at McGill University, Montreal, QC, Canada. His research interests include optical wireless communications and silicon photonics.

Lawrence R. Chen (S'95, SM'05) received the B.Eng. degree in electrical engineering and mathematics from McGill University, Montreal, QC, Canada in 1995 and the M.A.Sc. and $\mathrm{Ph} . \mathrm{D}$. degrees in electrical and computer engineering from the University of Toronto, Toronto, ON, Canada in 1997 and 2000, respectively. Since 2000, he has been with the Department of Electrical and Computer Engineering at McGill University. His research interests are in optical communications, silicon photonics, and microwave photonics, as well as engineering education and teaching pedagogy.

Dr. Chen is the Technical Program Chair of the 2019 IEEE International Topical Meeting on Microwave Photonics, a Guest Co-Editor of the IEEE/OSA Journal of Lightwave Technology Special Issue on Microwave Photonics (to be published in 2020), and the Principal Guest Editor of the IEEE Journal of Selected Topics in Quantum Electronics Issue on Optical Signal Processing (to be published in 2021). He is a Fellow of the Optical Society of America. 